\title{
ITWM
}

Fraunhofer

Institut

Techno- und

Wirtschaftsmathematik

K. Koch, J. Ohser, K. Schladitz

Spectral theory for random

closed sets and estimating

the covariance via frequency

space 
(c) Fraunhofer-Institut für Techno- und

Wirtschaftsmathematik ITWM 2002

\section{ISSN 1434-9973}

Bericht 37 (2002)

Alle Rechte vorbehalten. Ohne ausdrückliche, schriftliche Genehmigung des Herausgebers ist es nicht gestattet, das Buch oder Teile daraus in irgendeiner Form durch Fotokopie, Mikrofilm oder andere Verfahren zu reproduzieren oder in eine für Maschinen, insbesondere Datenverarbeitungsanlagen, verwendbare Sprache zu übertragen. Dasselbe gilt für das Recht der öffentlichen Wiedergabe.

Warennamen werden ohne Gewährleistung der freien Verwendbarkeit benutzt.

Die Veröffentlichungen in der Berichtsreihe des Fraunhofer ITWM können bezogen werden über:

Fraunhofer-Institut für Techno- und Wirtschaftsmathematik ITWM

Gottlieb-Daimler-Straße, Geb. 49

\section{Kaiserslautern}

Telefon: +49 (0) 6 31/2 05-32 42

Telefax: +49 (0) 6 31/2 05-4139

E-Mail: info@itwm.fraunhofer.de

Internet: www.itwm.fraunhofer.de 
Das Tätigkeitsfeld des Fraunhofer Instituts für Techno- und Wirtschaftsmathematik ITWM umfasst anwendungsnahe Grundlagenforschung, angewandte Forschung sowie Beratung und kundenspezifische Lösungen auf allen Gebieten, die für Techno- und Wirtschaftsmathematik bedeutsam sind.

In der Reihe »Berichte des Fraunhofer ITWM « soll die Arbeit des Instituts kontinuierlich einer interessierten Öffentlichkeit in Industrie, Wirtschaft und Wissenschaft vorgestellt werden. Durch die enge Verzahnung mit dem Fachbereich Mathematik der Universität Kaiserslautern sowie durch zahlreiche Kooperationen mit internationalen Institutionen und Hochschulen in den Bereichen Ausbildung und Forschung ist ein großes Potenzial für Forschungsberichte vorhanden. In die Berichtreihe sollen sowohl hervorragende Diplom- und Projektarbeiten und Dissertationen als auch Forschungsberichte der Institutsmitarbeiter und Institutsgäste zu aktuellen Fragen der Techno- und Wirtschaftsmathematik aufgenommen werden.

Darüberhinaus bietet die Reihe ein Forum für die Berichterstattung über die zahlreichen Kooperationsprojekte des Instituts mit Partnern aus Industrie und Wirtschaft.

Berichterstattung heißt hier Dokumentation darüber, wie aktuelle Ergebnisse aus mathematischer Forschungs- und Entwicklungsarbeit in industrielle Anwendungen und Softwareprodukte transferiert werden, und wie umgekehrt Probleme der Praxis neue interessante mathematische Fragestellungen generieren.

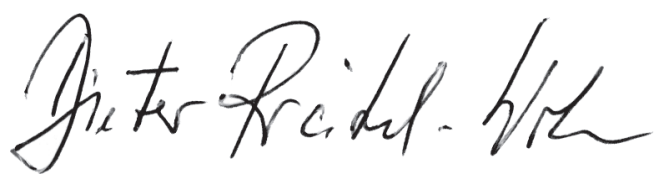

Prof. Dr. Dieter Prätzel-Wolters

Institutsleiter

Kaiserslautern, im Juni 2001 


\title{
SPECTRAL THEORY FOR RANDOM CLOSED SETS AND ES- TIMATING THE COVARIANCE VIA FREQUENCY SPACE
}

\author{
KARSTEN KOCH, ${ }^{*}$ Philipps University of Marburg \\ JOACHIM OHSER, KATJA SCHLADITZ, ${ }^{* *}$ Fraunhofer ITWM
}

\begin{abstract}
A spectral theory for stationary random closed sets is developed and provided with a sound mathematical basis. Definition and proof of existence of the Bartlett spectrum of a stationary random closed set as well as the proof of a Wiener-Khintchine theorem for the power spectrum are used to two ends: First, well known second order characteristics like the covariance can be estimated faster than usual via frequency space. Second, the Bartlett spectrum and the power spectrum can be used as second order characteristics in frequency space. Examples show, that in some cases information about the random closed set is easier to obtain from these characteristics in frequency space than from their real world counterparts.

Keywords: Random set, Bartlett spectrum, fast Fourier transform, power spectrum
\end{abstract}

AMS 2000 Subject Classification: Primary 60D05

Secondary $62 \mathrm{G} 05,42 \mathrm{~B} 10,62 \mathrm{M} 40$

\section{Introduction}

Random closed sets (RACS) are an important class of models in stochastic geometry, used to describe such diverse structures as microstructures in materials science, patches of vegetation, territories of animals, or ore deposits. Stationarity - invariance of the distribution with respect to translations - is usually assumed. Popular and powerful

* Postal address: Philipps University of Marburg, Faculty of Mathematics and Informatics, HansMeerwein Str., Lahnberge, D-35032 Marburg, Germany

** Postal address: Fraunhofer ITWM, Gottlieb-Daimler-Str.49, D-67663 Kaiserslautern, Germany 
tools for describing stationary random closed sets are second order characteristics, in particular covariance, covariance function, and covariogram.

There is a close connection between stochastic geometry and image analysis as data sets which can be modeled as random closed sets are given as digital images. Thus it is natural to use tools from image analysis to estimate quantities from stochastic geometry. For example, the Euclidean distance transform is used to estimate the empty space function. However, spectral analysis has been sidelined so far although it plays a central role in image analysis (see [9]).

Some imaging techniques based on diffraction (for instance of x-rays) yield characteristics in frequency space [3]. These characteristics are related to quantities in real space as (co)variograms and correlation functions via the Fourier transform [9]. The fast Fourier transform (FFT) and sophisticated algorithms for its computation $[10,14]$ allow to determine these quantities fast and efficiently. In some cases however, the second order characteristics in frequency space are easier to describe and interpret $[17,24]$.

In stochastic geometry, spectral analysis is mainly used for point processes. See $[1,2]$ for the theoretic foundations for one and two dimensional point processes and [18] and [15] for applications to ecological data. More general approaches can be found in [6, Chapter 11] and [19]. In [6, Chapter 11] Daley and Vere-Jones generalize Bartlett's theory $[1,2]$ to stationary random measures with an additional invariance property. Ripley develops in [19] the spectral analysis for general spatial random processes.

The spectral approach and thus the fast Fourier transform can be used to create estimators for second order characteristics like the covariance being faster than those usually used in stochastic geometry. More precisely, we suggest estimating the covariance $C_{0}$ of a stationary RACS $\Xi$ observed in a compact window $W$ using the right side of the following diagram:

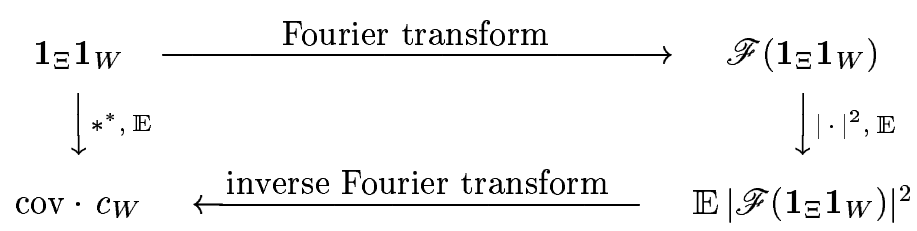

Here 1 denotes the characteristic function and $c_{W}$ is the set covariance of $W$. 
This idea has been around for some time [7], see also [16, Chapter 5]. However, to our knowledge it has never been made rigorous. Therefore the aim of this paper is twofold. First, we provide a sound mathematical basis for this way of estimating the covariance and more generally for the spectral analysis of stationary random closed sets. The main steps to achieve this are to prove the existence of the Bartlett spectrum and an equivalent of the Wiener-Khintchine theorem for the power spectrum of $\Xi$ w.r.t. $W$, which is closely related to the quantity in the lower right corner of the diagram. Second, we suggest the use of the Bartlett spectrum or of the power spectrum as characteristics in frequency space for the description of stationary random closed sets.

This paper is is based on the master's thesis [12] of the first author. It is organized as follows: After providing some basic concepts in the next section, we define and prove the existence of the Bartlett spectrum in Section 3. Proving an equivalent of the Wiener-Khintchine theorem for the power spectrum of $\Xi$ w.r.t. $W$ in Section 4 provides the basis for estimating the covariance via frequency space. This estimation procedure is described in Section 5. The complexity of the proposed algorithm and the overlapping effect are discussed in this section, too. Section 6 is devoted to the special cases of isotropic RACS in $\mathbb{R}^{2}$ and $\mathbb{R}^{3}$. Bartlett spectrum and covariance are estimated for both simulated and real data sets in Section 7. For some of the examples, the Bartlett spectrum turns out to be much easier to interpret than the covariance.

\section{Preliminaries}

Let $\Xi$ denote a $d$-dimensional random closed set (RACS) in terms of [21]. Let $C$ be the covariance and let cov be the covariance function of $\Xi$. If $\Xi$ is stationary then both functions depend on the difference of their arguments, only, and shall be denoted by $C_{0}$ and $\operatorname{cov}_{0}$, respectively.

For an integrable function $f: \mathbb{R}^{d} \rightarrow \mathbb{C}$ the Fourier transform $\mathscr{F}$ is defined by

$$
\mathscr{F} f(\omega):=\widehat{f}(\omega):=\frac{1}{\sqrt{2 \pi}^{d}} \int_{\mathbb{R}^{d}} f(x) e^{-i\langle x, \omega\rangle} d x, \quad \omega \in \mathbb{R}^{d} .
$$

The inverse Fourier transform $\overline{\mathscr{F}}$ is defined similarly by replacing $-i$ with $i$. In the literature these transforms are defined in various ways. Although generating basically the same results, the usage of different definitions can lead to different scaled functions. 
This is important to observe especially when computing a discrete version of the Fourier transform. In the following an extension of the Fourier transform to measures will be used. A measure $\nu$ shall be denoted as the Fourier transform of the measure $\mu$, if for all $\psi \in \mathscr{S}\left(\mathbb{R}^{d}\right)$, i.e. functions of rapid decay, the Parseval identity

$$
\int_{\mathbb{R}^{d}} \psi(x) \nu(d x)=\int_{\mathbb{R}^{d}} \widehat{\psi}(\omega) \mu(d \omega)
$$

holds. In this context the following theorem is very useful.

Theorem 1. (Bochner.) For any continuous and positive definite function $f: \mathbb{R}^{d} \rightarrow$ $\mathbb{C}$ there exists a unique positive finite Radon measure $\mu$ with

$$
f(x)=\frac{1}{\sqrt{2 \pi}^{d}} \int_{\mathbb{R}^{d}} e^{i\langle\omega, x\rangle} \mu(d \omega), \quad x \in \mathbb{R}^{d} .
$$

For the proofe see [8, Chapter II.3].

\section{Spectrum}

In this section we prove the existence of a second order characteristic of stationary RACS in frequency space. In general, neither the covariance $C_{0}$ nor the covariance function $\operatorname{cov}_{0}$ of a $d$-dimensional stationary RACS are elements of $L^{p}\left(\mathbb{R}^{d}\right), 1 \leq p \leq$ 2, therefore their Fourier transforms are not defined and another approach must be chosen. It is advantageous to introduce the covariance measure $\mathrm{Cov}_{0}$ of a RACS, which is defined by

$$
\operatorname{Cov}_{0}(A):=\int_{A} \operatorname{cov}_{0}(x) d x, \quad \text { for any Borel set } A .
$$

Our proof is essentially a special case of the one given in [6, Chapter 11]. We identify $\mathrm{Cov}_{0}$ as the reduced covariance measure associated with the random volume measure induced by the RACS, which has a Fourier transform in the above sense.

For a $d$-dimensional stationary RACS $\Xi$ with positive volume density the volume measure $V_{\Xi}$, defined by

$$
V_{\Xi}(A):=V_{d}(A \cap \Xi) \text { for any bounded Borel set } A \subset \mathbb{R}^{d},
$$


is a stationary random measure, which is finite for any bounded Borel set. For bounded Borel sets $A, B \subset \mathbb{R}^{d}$ we define a measure

$$
\operatorname{Cov} \Xi(A, B):=\operatorname{cov}\left(V_{\Xi}(A), V_{\Xi}(B)\right)
$$

which satisfies the well-known relation

$$
\operatorname{Cov} \Xi(A, B)=\int_{\mathbb{R}^{d}} V_{d}\left(A \cap B_{h}\right) \operatorname{Cov}_{0}(d h),
$$

see e.g. [23, Chapter 7.3.4]. This can be generalized as follows. Assume $f: \mathbb{R}^{d} \times \mathbb{R}^{d} \rightarrow$ $\mathbb{R}$ is a bounded measurable function with compact support. Then Fubini's theorem yields

$$
\int_{\mathbb{R}^{d} \times \mathbb{R}^{d}} f(x, y) \operatorname{Cov} \Xi(d x, d y)=\int_{\mathbb{R}^{d}} \int_{\mathbb{R}^{d}} f(x, x+h) d x \operatorname{Cov}_{0}(d h) .
$$

An analogous result holds for the covariance of the volume measure and the covariance of the RACS $\Xi$. In terms of [6, Definition 10.4.I. and Lemma 10.4.III] this means that the volume measure $V_{\Xi}$ is a second-order stationary random measure and that $\operatorname{Cov}_{0}$ is the reduced covariance measure with respect to $V_{\Xi}$.

By Proposition 11.2.I. in [6] there exists a locally finite measure $\Gamma$ associated with the measure $\mathrm{Cov}_{0}$ by the Parseval relation (1)

$$
\int_{\mathbb{R}^{d}} \widehat{\psi}(x) \operatorname{Cov}_{0}(d x)=\int_{\mathbb{R}^{d}} \psi(\xi) \Gamma(d \xi)
$$

for any $\psi \in \mathscr{S}\left(\mathbb{R}^{d}\right)$. Thus $\Gamma$ is the Fourier transform of $\operatorname{Cov}_{0}$ and is called the Bartlett spectrum of $\Xi$.

This already proves the existence of a characteristic in frequency space but until now one important property of the measure $\mathrm{Cov}_{0}$ has been omitted: the covariance function $\operatorname{cov}_{0}$ is continuous, therefore some nice properties of the Bartlett spectrum can be expected. This is specified in the following theorem, which also implies the existence of the Bartlett spectrum. The continuity of the covariance function was proved by Heinrich, see $[4,12]$.

Theorem 2. For any stationary RACS $\Xi$ with positive volume fraction the Bartlett spectrum $\Gamma$ is a totally finite Radon measure, i.e. there exists a totally finite Radon 
measure $\Gamma$ such that

$$
\int_{\mathbb{R}^{d}} \widehat{\psi}(x) \operatorname{cov}_{0}(x) d x=\int_{\mathbb{R}^{d}} \psi(\omega) \Gamma(d \omega)
$$

for all $\psi \in \mathscr{S}\left(\mathbb{R}^{d}\right)$.

Proof. Let $p$ be the volume fraction of $\Xi$ and let $\varphi: \mathbb{R}^{d} \rightarrow \mathbb{C}$ be a continuous function with compact support. Since the covariance function $\operatorname{cov}_{0}$ is continuous and bounded the following integral exists and Fubini's theorem yields

$$
\begin{aligned}
& \int_{\mathbb{R}^{d}} \int_{\mathbb{R}^{d}} \varphi(x) \overline{\varphi(y)} \operatorname{cov}_{0}(y-x) d x d y \\
& \quad=\mathbb{E} \int_{\mathbb{R}^{d}} \int_{\mathbb{R}^{d}} \varphi(x) \overline{\varphi(y)}\left(\mathbf{1}_{\Xi}(x)-p\right)\left(\mathbf{1}_{\Xi}(y)-p\right) d x d y \\
& \quad=\mathbb{E}\left|\int_{\mathbb{R}^{d}} \varphi(x)\left(\mathbf{1}_{\Xi}(x)-p\right) d x\right|^{2} \geq 0 .
\end{aligned}
$$

This is equivalent to the positive definiteness of $\operatorname{cov}_{0}$, see [20, Theorem 1.7.3]. By Bochner's theorem (e.g. [8, Chapter 2, Theorem 3.6]) there exists a totally finite Radon measure $\Gamma$ with

$$
\operatorname{cov}_{0}(x)=\frac{1}{\sqrt{2 \pi}^{d}} \int_{\mathbb{R}^{d}} e^{i\langle\omega, x\rangle} \Gamma(d \omega) .
$$

Again, for any $\psi \in \mathscr{S}\left(\mathbb{R}^{d}\right)$, Fubini's theorem yields Equation (4).

\section{A Wiener-Khintchine theorem}

By the theorem of Wiener-Khintchine (e.g. [13, p. 299]) the power spectrum of a signal coincides with the Fourier transform of its autocorrelation function. This is essentially a special case of the well known convolution theorem from Fourier analysis. A similar result can be established for stationary RACS and the covariance function. This leads to a new estimator for the covariance.

Let $\Xi$ be a stationary $d$-dimensional RACS with positive volume fraction $p$. Due to the unboundedness of any stationary RACS the random process defined by the characteristic function of $\Xi$ is almost surely not Fourier transformable. Therefore it must be restricted to a compact window $W \subset \mathbb{R}^{d}$. The random function

$$
\xi_{W}(x):=\mathbf{1}_{W}(x)\left(\mathbf{1}_{\Xi}(x)-p\right), \quad x \in \mathbb{R}^{d},
$$


is a.s. integrable, thus we can define the power spectrum of $\Xi$ with respect to $W$ by

$$
\operatorname{pow}_{W}(\omega):=\mathbb{E}\left|\widehat{\xi}_{W}(\omega)\right|^{2}, \quad \omega \in \mathbb{R}^{d} .
$$

For all $\omega \in \mathbb{R}^{d}$ we have

$$
\begin{aligned}
\operatorname{pow}_{W}(\omega) & =\mathbb{E} \mathscr{F} \xi_{W}(\omega) \overline{\mathscr{F}} \xi_{W}(\omega) \\
& =\frac{1}{(2 \pi)^{d}} \mathbb{E} \int_{\mathbb{R}^{d}} \int_{\mathbb{R}^{d}} \mathbf{1}_{W}(x) \mathbf{1}_{W}(y)\left(\mathbf{1}_{\Xi}(x)-p\right)\left(\mathbf{1}_{\Xi}(y)-p\right) e^{-i\langle x-y, \omega\rangle} d y d x \\
& =\frac{1}{(2 \pi)^{d}} \int_{\mathbb{R}^{d}} \int_{\mathbb{R}^{d}} \mathbf{1}_{W}(x) \mathbf{1}_{W}(y) e^{-i\langle x-y, \omega\rangle} \operatorname{cov}_{0}(x-y) d y d x .
\end{aligned}
$$

Defining the set covariance of $W$ by

$$
c_{W}(x):=\int_{\mathbb{R}^{d}} \mathbf{1}_{W}(y) \mathbf{1}_{W}(y-x) d y, \quad x \in \mathbb{R}^{d},
$$

we obtain

$$
\operatorname{pow}_{W}(\omega)=\frac{1}{(2 \pi)^{d}} \int_{\mathbb{R}^{d}} c_{W}(h) e^{-i\langle h, \omega\rangle} \operatorname{cov}_{0}(h) d h .
$$

This proves the following analogue to the theorem of Wiener-Khintchine.

Theorem 3. Let $\Xi$ be a stationary $R A C S$ and $W \subset \mathbb{R}^{d}$ be compact, then

$$
\sqrt{2 \pi}^{d} \operatorname{pow}_{W}(\omega)=\mathscr{F}\left(c_{W} \cdot \operatorname{cov}_{0}\right)(\omega)
$$

holds for all $\omega \in \mathbb{R}^{d}$.

By this theorem a representation of the Bartlett spectrum $\Gamma$ can be obtained. Since the set covariance $c_{W}$ has compact support, the right hand side of equation (6) is finite for all $\omega \in \mathbb{R}^{d}$. Equation (5) yields

$$
\begin{aligned}
\int_{\mathbb{R}^{d}} c_{W}(x) e^{-i\langle x, \omega\rangle} \operatorname{Cov}_{0}(d x) & =\int_{\mathbb{R}^{d}} c_{W}(x) e^{-i\langle x, \omega\rangle} \frac{1}{\sqrt{2 \pi}^{d}} \int_{\mathbb{R}^{d}} e^{i\langle x, \zeta\rangle} \Gamma(d \zeta) d x \\
& =\int_{\mathbb{R}^{d}} \frac{1}{\sqrt{2 \pi}^{d}} \int_{\mathbb{R}^{d}} c_{W}(x) e^{-i\langle x, \omega-\zeta\rangle} d x \Gamma(d \zeta) \\
& =\int_{\mathbb{R}^{d}} \widehat{c}_{W}(\omega-\zeta) \Gamma(d \zeta) .
\end{aligned}
$$

Corollary 1. The Bartlett spectrum $\Gamma$ of $\Xi$ can be represented as

$$
(2 \pi)^{d} \operatorname{pow}_{W}(\omega)=\left(\widehat{c}_{W} * \Gamma\right)(\omega), \quad \omega \in \mathbb{R}^{d} .
$$




\section{Estimation of the covariance}

A straight forward approach for the estimation of the covariance of $\Xi$ observed in a compact window $W$ is given by the relation

$$
C_{0}(h)=\mathbb{E} \frac{V((\Xi \cap W) \cap((\Xi \cap W)-h))}{c_{W}(h)}
$$

for all $h \in \mathbb{R}^{d}$ with $c_{W}(h)>0$, see [16, p. 150]. Given a sample $\Xi_{0}$ of $\Xi$ on a lattice $\triangle \mathbb{Z}^{n}, \triangle>0$, in $W$ with $N=\left|\Xi_{0} \cap \triangle \mathbb{Z}^{n} \cap W\right|$ the covariance can be computed with a complexity in $O\left(N^{2}\right)$.

A faster computable estimator can be obtained by Theorem 3. Substitute $\xi_{W}$ by the random function

$$
g_{W}(x):=\mathbf{1}_{W}(x) \mathbf{1}_{\Xi}(x), \quad x \in \mathbb{R}^{d} .
$$

Analogous to Theorem 3 we obtain

$$
\sqrt{2 \pi}^{d} \mathbb{E}\left|\widehat{g}_{W}\right|^{2}=\mathscr{F}\left(c_{W} \cdot C_{0}\right) .
$$

Since $g_{W} \in L^{1}\left(\mathbb{R}^{d}\right) \cap L^{2}\left(\mathbb{R}^{d}\right)$ a.s., the function $\mathbb{E}\left|\widehat{g}_{W}\right|^{2}$ is integrable and the inverse Fourier transform can be applied

$$
\sqrt{2 \pi}^{d} \overline{\mathscr{F}}\left(\mathbb{E}\left|\widehat{g}_{W}\right|^{2}\right)=c_{W} \cdot C_{0} .
$$

Assume $0 \in \stackrel{\circ}{W}$, where $\stackrel{\circ}{W}$ denotes the interior of $W$, then $c_{W}$ is positive for all $h \in \stackrel{\circ}{W}$ and for all such $h$ Fubini's theorem yields

$$
C_{0}(h)=\mathbb{E} \frac{\sqrt{2 \pi}^{d}\left(\overline{\mathscr{F}}\left|\widehat{g}_{W}\right|^{2}\right)(h)}{c_{W}(h)} .
$$

A discretization of this equation gives an estimator for the covariance.

For a sample of $\Xi$ consisting of $N$ points the covariance can be computed by the use of the Fast Fourier Transform in a complexity in $O(N \log N)$. This holds, because the FFT has a complexity in $O(N \log N)$ and the set covariance of $W$ can be computed as $c_{W}=\overline{\mathscr{F}}\left|\mathscr{F} \mathbf{1}_{W}\right|^{2}$.

Unfortunately the assumption of periodicity within the FFT causes an overlapping effect, see [5, Chapter 6.4]. In the following this effect is illustrated in the onedimensional case. The FFT is only a special case of the discrete Fourier transform 
(DFT, see [5, Chapter 6.2]), therefore the DFT is used. Assume $W:=\left[-\frac{T}{2},(N-\right.$ 1) $\left.T+\frac{T}{2}\right]$ with $T \in \mathbb{R}^{+}$and assume $N$ sample points

$$
h_{k}:=k T, \quad k \in\{0, \ldots, N-1\}
$$

For a function $g: W \cap T \mathbb{Z} \rightarrow \mathbb{R}$ and $m \in\{0, \ldots, N-1\}$ we have

$$
\begin{aligned}
\left|\operatorname{DFT}(g)\left(\frac{m}{N T}\right)\right|^{2} & =\left(\sum_{k=0}^{N-1} g(k T) e^{-i 2 \pi \frac{k m}{N}}\right)\left(\sum_{l=0}^{N-1} g(l T) e^{i 2 \pi \frac{l m}{N}}\right) \\
& =\sum_{k=0}^{N-1} \sum_{l=0}^{N-1} g(k T) g(l T) e^{-i 2 \pi \frac{(k-l) m}{N}} .
\end{aligned}
$$

Applying the inverse DFT (iDFT, [5, Chapter 6.3]) we get

$$
\begin{aligned}
i D F T\left(|D F T(g)|^{2}\right)\left(h_{k}\right) & =\frac{1}{N} \sum_{m=0}^{N-1}\left|D F T(g)\left(\frac{m}{N T}\right)\right|^{2} e^{i 2 \pi \frac{k m}{N}} \\
& =\frac{1}{N} \sum_{n=0}^{N-1} \sum_{l=0}^{N-1} g(n T) g(l T) \sum_{m=0}^{N-1} e^{-i 2 \pi m \frac{n-l-k}{N}} \\
& =\sum_{l=0}^{N-1} g(l T) g(((l+k) \bmod N) T) .
\end{aligned}
$$

Substituting $\mathbf{1}_{\Xi_{0} \cap \mathbb{Z} \cap W}$ for $g$ with a sample $\Xi_{0}$ of $\Xi$ we obtain

$$
\sum_{l=0}^{N-1} \mathbf{1}_{\Xi_{0}}(l T) \mathbf{1}_{\Xi_{0}}(((l+k) \bmod N) T)
$$

as the discretization of the numerator in Equation (8). The corresponding discretization of the numerator in Equation (7) is

$$
\sum_{l=0}^{N-1} \mathbf{1}_{\Xi_{0}}(l T) \mathbf{1}_{\Xi_{0} \cap W}((l+r) T) .
$$

Hence the overlapping effect leads to counting the lattice points in $\Xi_{0} \cap W \cap\left(\widetilde{\Xi}_{0}-r T\right)$, where $\widetilde{\Xi}_{0}$ denotes the $W$-periodic continuation of $\Xi$, instead of counting the lattice points in $\left(\Xi_{0} \cap W\right) \cap\left(\left(\Xi_{0} \cap W\right)-r T\right)$.

The effect can be eliminated by expanding the function $g$ to the window $2 W$ :

$$
g_{2}(h)= \begin{cases}g(h) & \text { if } h \in W, \\ 0 & \text { if } h \in 2 W \backslash W .\end{cases}
$$


In the $\mathbb{R}^{d}$-case this increases the number of sample points to $2^{d} N$. Still the complexity belongs to $O(N \log N)$. This is a considerable gain compared to the usual estimation of the covariance with the complexity $O\left(N^{2}\right)$.

\section{The isotropic case}

Now consider a stationary RACS $\Xi$ in $\mathbb{R}^{d}$ which in addition is isotropic. Its covariance function is rotationally symmetric, i.e. $\operatorname{cov}_{0}(h)=: \overline{\operatorname{cov}}_{0}(\|h\|)$. The symmetry properties of the power spectrum depend on the set covariance of $W$. Therefore a suitable set $W$ must be chosen to obtain also rotation symmetry for the power spectrum. This can always be done by choosing a $d$-dimensional ball $b_{d}(R)$ with radius $R>0$.

case: $d=1$

The set covariance $c_{W}$ is symmetric for all compact $W$, therefore Equation (6) yields for $\omega \geq 0$

$$
\begin{aligned}
\operatorname{pow}_{W}(\omega) & =\frac{1}{2 \pi} \int_{\mathbb{R}} c_{W}(h) \operatorname{cov}_{0}(h) e^{-i h \omega} d h \\
& =\frac{1}{\pi} \int_{0}^{\infty} c_{W}(h) \overline{\operatorname{cov}}_{0}(h)\left(e^{-i h \omega}+e^{i h \omega}\right) d h \\
& =\frac{2}{\pi} \int_{0}^{\infty} c_{W}(h) \overline{\operatorname{cov}}_{0}(h) \cos (h \omega) d h .
\end{aligned}
$$

So the covariance function is connected to the power spectrum by the (inverse) cosine transformation.

case: $d=2$

In the two-dimensional case an arbitrary window $W$ can be chosen. A simplification of Equation (6) can be obtained by considering the rotation mean of the power spectrum, using polar coordinates i.e.

$$
\overline{\operatorname{pow}}_{W}(\rho)=\frac{1}{2 \pi} \int_{0}^{2 \pi} \operatorname{pow}_{W}(\rho \cos \phi, \rho \sin \phi) d \phi, \quad \rho \geq 0 .
$$


By Equation (6) it follows that

$$
\begin{aligned}
& \overline{\operatorname{pow}}_{W}(\rho) \\
& =\frac{1}{(2 \pi)^{3}} \int_{0}^{2 \pi} \int_{\mathbb{R}} \int_{\mathbb{R}} c_{W}(x, y) \operatorname{cov}_{0}(x, y) e^{-i \rho(x \cos \phi+y \sin \phi)} d x d y d \phi \\
& =\frac{1}{(2 \pi)^{3}} \int_{0}^{2 \pi} \int_{0}^{\infty} \int_{0}^{2 \pi} r \overline{\operatorname{cov}}_{0}(r) c_{W}(r \cos \varphi, r \sin \varphi) e^{-i r \rho(\cos \varphi \cos \phi+\sin \varphi \sin \phi)} d \varphi d r d \phi \\
& =\frac{1}{(2 \pi)^{2}} \int_{0}^{\infty} r \overline{\operatorname{cov}}_{0}(r) \int_{0}^{2 \pi} c_{W}(r \cos \varphi, r \sin \varphi) \frac{1}{2 \pi} \int_{0}^{2 \pi} e^{i r \rho \cos (\phi-\varphi+\pi)} d \phi d \varphi d r .
\end{aligned}
$$

Since the inner integral is just the Bessel function of order zero, i.e.

$$
\mathscr{J}_{0}(x)=\frac{1}{2 \pi} \int_{0}^{2 \pi} e^{i x \cos \vartheta} d \vartheta, \quad x \geq 0,
$$

it follows that

$$
\begin{aligned}
\overline{\operatorname{pow}}_{W}(\rho) & =\frac{1}{(2 \pi)^{2}} \int_{0}^{\infty} r \overline{\operatorname{cov}}_{0}(r) \mathscr{J}_{0}(r \rho) \int_{0}^{2 \pi} c_{W}(r \cos \varphi, r \sin \varphi) d \varphi d r \\
& =\frac{1}{2 \pi} \int_{0}^{\infty} r \overline{\operatorname{cov}}_{0}(r) \bar{c}_{W}(r) \mathscr{J}_{0}(r \rho) d r .
\end{aligned}
$$

The power spectrum is therefore the Bessel transform of the function $\bar{c}_{W} \cdot \overline{\operatorname{cov}}_{0}$, see $[6$, p. 449]. Its inverse transform can be obtained by substituting the factor $(2 \pi)^{-1}$ by $2 \pi$. Without these factors the transform is also known as the Hankel transform (e.g. [8, p. $130 \mathrm{ff}])$.

\section{case: $d=\mathbf{3}$}

In the three-dimensional case a spherical set $W$ must be chosen to achieve a simplification of Equation (6). Let the $(x, y, z)$-space and the transformed $(u, v, w)$-space be represented by polar coordinates, i.e.

$$
\begin{aligned}
x & =r \cos \varphi \sin \vartheta, & u & =\rho \cos \phi \sin \theta, \\
y & =r \sin \varphi \sin \vartheta, & v & =\rho \sin \phi \sin \theta, \\
z & =r \cos \vartheta, & w & =\rho \cos \theta,
\end{aligned}
$$


with $(r, \vartheta, \varphi),(\rho, \theta, \phi) \in[0, \infty) \times[0, \pi] \times[0,2 \pi)$. For the inner product it follows, that

$$
\langle(x, y, z),(u, v, w)\rangle=r \rho(\cos (\varphi-\phi) \sin \vartheta \sin \theta+\cos \vartheta \cos \theta) .
$$

Now with $W:=b_{3}(R), R>0$, the power spectrum is rotationally symmetric. A proper choice of the parameters $\theta$ and $\phi$ yields

$$
\begin{aligned}
\overline{\operatorname{pow}}_{W}(\rho) & =\operatorname{pow}_{W}(\rho, \theta, \phi)=\operatorname{pow}_{W}(\rho, 0, \phi) \\
& =\frac{1}{(2 \pi)^{3}} \int_{0}^{\infty} \int_{0}^{\pi} \int_{0}^{2 \pi} \bar{c}_{W}(r) \overline{\operatorname{cov}}_{0}(r) r^{2} \sin \vartheta e^{-i r \rho \cos \vartheta} d \varphi d \vartheta d r \\
& =\frac{1}{(2 \pi)^{2}} \int_{0}^{\infty} \bar{c}_{W}(r) \overline{\operatorname{cov}}_{0}(r) r^{2} \int_{0}^{\pi} \sin \vartheta e^{-i r \rho \cos \vartheta} d \vartheta d r .
\end{aligned}
$$

With

$$
\int_{0}^{\pi} \sin \vartheta e^{-i r \rho \cos \vartheta} d \vartheta=\frac{2}{r \rho} \sin (r \rho)
$$

it follows that

$$
\overline{\operatorname{pow}}_{W}(\rho)=\frac{1}{2 \pi^{2} \rho} \int_{0}^{\infty} r \bar{c}_{W}(r) \overline{\operatorname{cov}}_{0}(r) \sin (r \rho) d r, \quad \rho \geq 0 .
$$

So the function $r \bar{c}_{W}(r) \overline{\operatorname{cov}}_{0}(r)$ can be obtained by applying the inverse sine transform to $\rho \overline{\operatorname{pow}}_{W}(\rho)$. Formulas of this kind play an significant role in the theory of small angle scattering (see [7]).

In these cases isotropy leads to a remarkable simplification of the practical calculation of the covariance function. Instead of a full-dimensional inverse Fourier transform, simply a one-dimensional special case of the Fourier transform can be applied. This can reduce the computation time substantially. Unfortunately, in the three-dimensional case, where immense amounts of data can occur, one is restricted to an unusual spherical window $W$.

\section{Examples}

In this section the covariances and densities of Bartlett spectra of samples of two- and three-dimensional RACS are estimated. The underlying RACS are either simulated or 


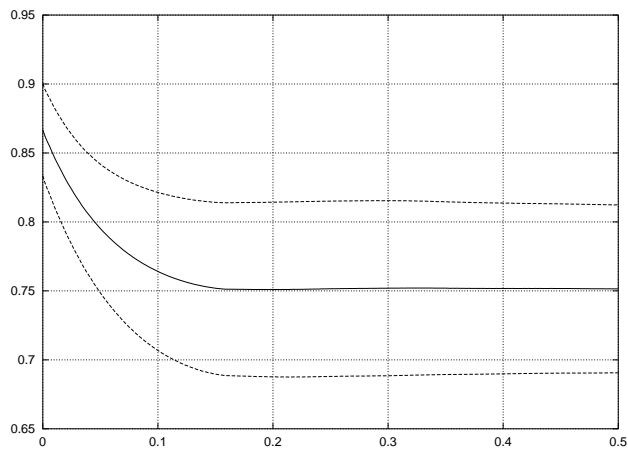

(a)

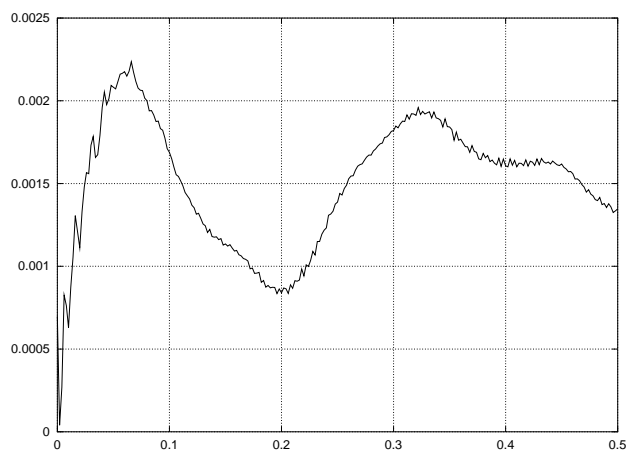

(b)

Figure 1: (a) Rotation mean and standard deviation (dashed) of the estimated covariance of a two-dimensional Boolean model. (b) Deviation of the estimated covariance from the theoretical covariance.

correspond to real structures. Of the real structures only small probes are given, therefore, assuming macroscopic homogeneity, they are interpreted as stationary RACS. For interpretability only the rotation means of the above functions are displayed.

Example 1. We consider an isotropic two-dimensional Boolean model with constant discs of radius $R=0.08$. It is based on a Poisson process with intensity $\lambda=100$. The model is simulated in a square of side length 1 , dicretized by $500 \times 500$ lattice points.

Figure 1a shows the rotation mean of the estimated covariance averaged over 100 simulations. Additionally, the sum and difference of the estimated covariance and its standard deviation are plotted. Figure $1 \mathrm{~b}$ shows the deviation of the estimated covariance from the theoretical covariance of the Boolean model.

Example 2. Figure 2 shows a planar section through an eutectic aluminium-nickel alloy. The $\alpha$-phase (dark constituent) can be interpreted as a realization of a twodimensional stationary RACS. The rotation mean of the estimated covariance (Figure 3a) of the $\alpha$-phase is oscillating with a rapidly declining amplitude, therefore interpreting the oscillation is rather difficult. The estimated density of the RACS's Bartlett spectrum (Figure $3 \mathrm{~b}$ ) has a single peak, thus the oscillation of the covariance corresponds to only one characteristic frequency/wavelength $\rho_{0}$. The inverse $r_{0}=1 / \rho_{0} \approx 2.1 \mu \mathrm{m}$ of the characteristic frequency is the position of the first local 


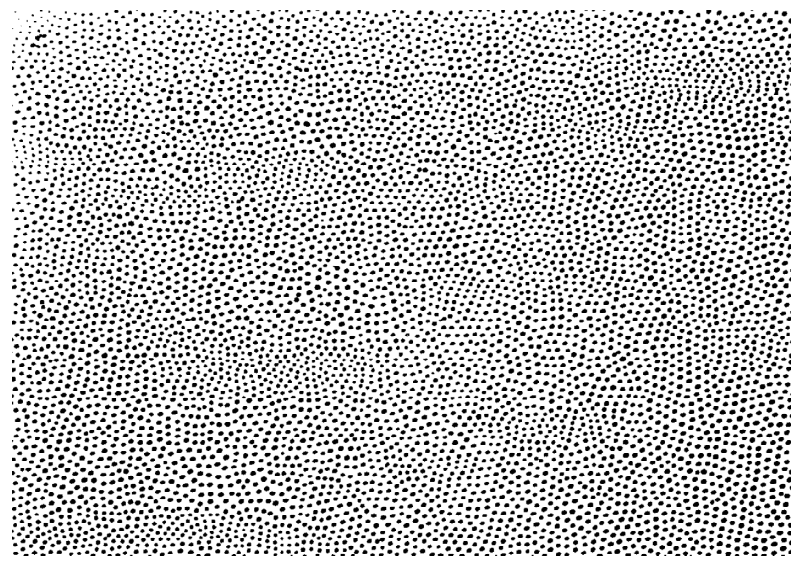

Figure 2: Planar section through an eutectic Al-Ni alloy of physical size $0.20 \mathrm{~mm} \times 0.14$ $\mathrm{mm}(1280 \times 896$ pixels $)$. The cut is perpendicular to the main direction of the needle-shaped $\alpha$-particles.

maximum of the covariance function. It corresponds to the characteristic nearest neighbor distance of the centers of the section profiles of the $\alpha$-needles.

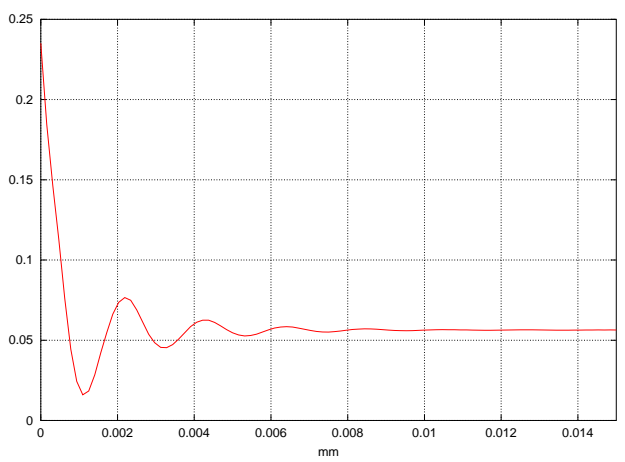

(a) Covariance

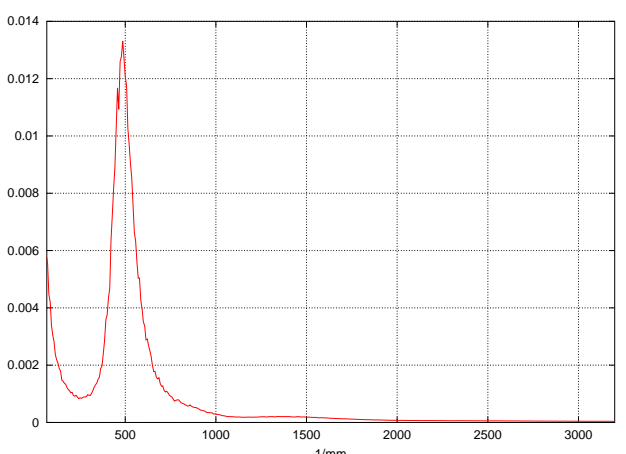

(b) Density of the Bartlett spectrum

FigURE 3: Rotation mean of estimated covariance and the estimated density of the Bartlett spectrum of the dark constituent of the Al-Ni alloy

Example 3. Figure 4 shows a visualization of a three-dimensional image of an open nickel foam. The solid constituent of the foam is interpreted as a three-dimensional stationary RACS. Its covariance (Figure 5) shows a local maximum at $0.4 \mathrm{~mm}$ which corresponds to the typical cell width of the foam. We remark that the position of a 


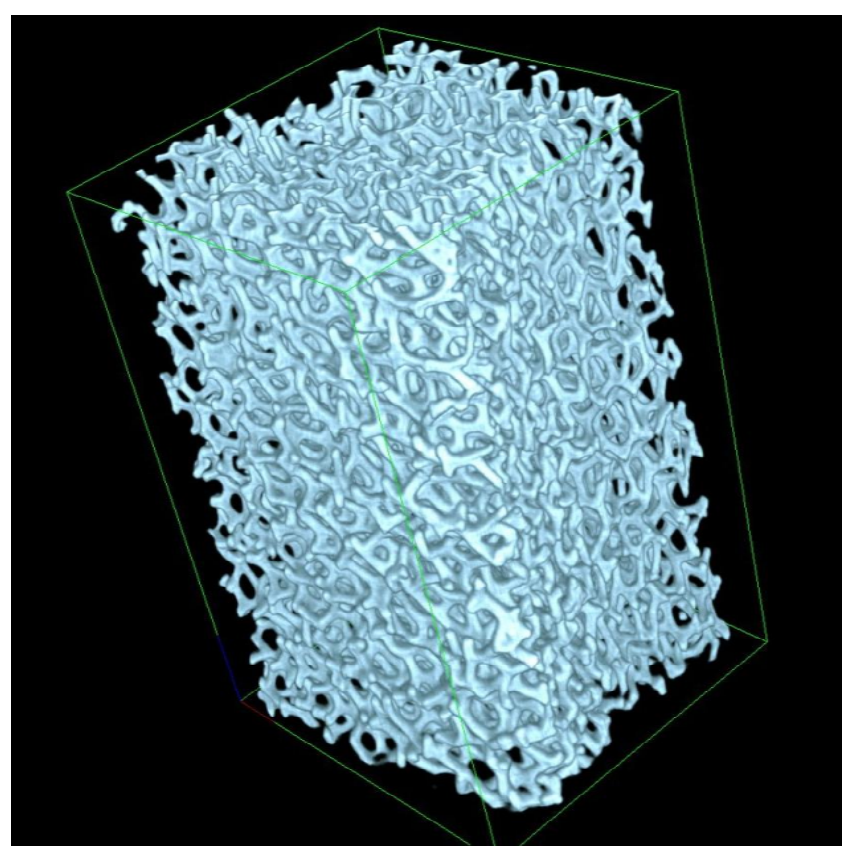

Figure 4: A visualization of a three-dimensional image of an open nickel foam. The specimen is of physical size $3.41 \mathrm{~mm} \times 3.21 \mathrm{~mm} \times 5.11 \mathrm{~mm}(256 \times 256 \times 256$ pixels $)$. The image was taken by x-ray computer tomography (XCT).

local maximum in the covariance function is highly independent of the conditions for image acquisition. This measuring the typical cell width using the estimation of the covariance function is robust with respect to modifications of the applied micro-focus $\mathrm{x}$-ray source, the sensitivity of the detector, the CT reconstruction algorithm, etc.

Example 4. Finally, we consider random packings of identical balls. Until now there exist no mathematical models for random packings. Their distribution properties depend on the algorithm applied to simulate samples. Here we restrict ourselves to the so-called force based algorithm, see [22] and references therein. The sample shown in Figure 6 was simulated in the unit cube, the volume density is $p=0.64$. The force based algorithm was modified such that the sample is periodic w.r.t. the unit cube. Therefore, the union of the balls can be considered as a realization of a stationary random set.

The covariance and the density of the Bartlett spectrum are shown in Figures $7 \mathrm{a}$ and 7b, respectively. Analogously to Example 2, the balls are arranged very regular. 


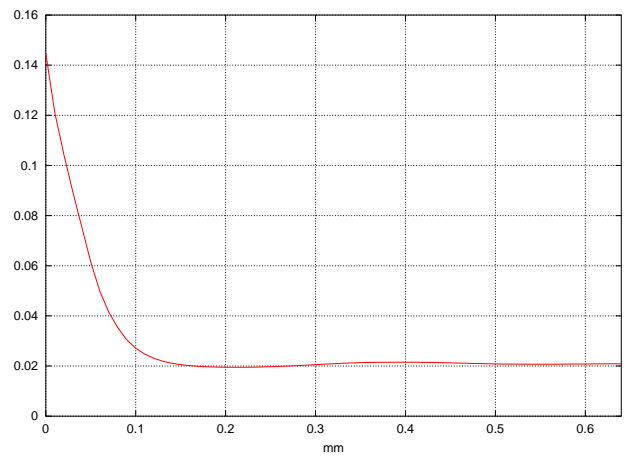

(a) Covariance

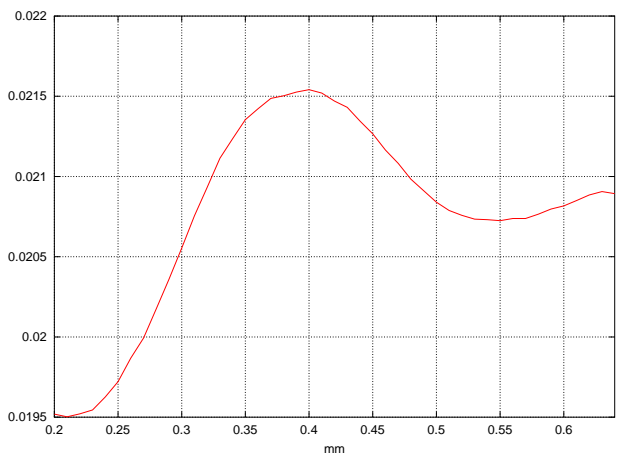

(b) Detail

FigURE 5: Rotation mean of the estimated covariance regarding a sample of the open nickel foam show in Figure 4. The right diagram is a magnified detail of the left one.

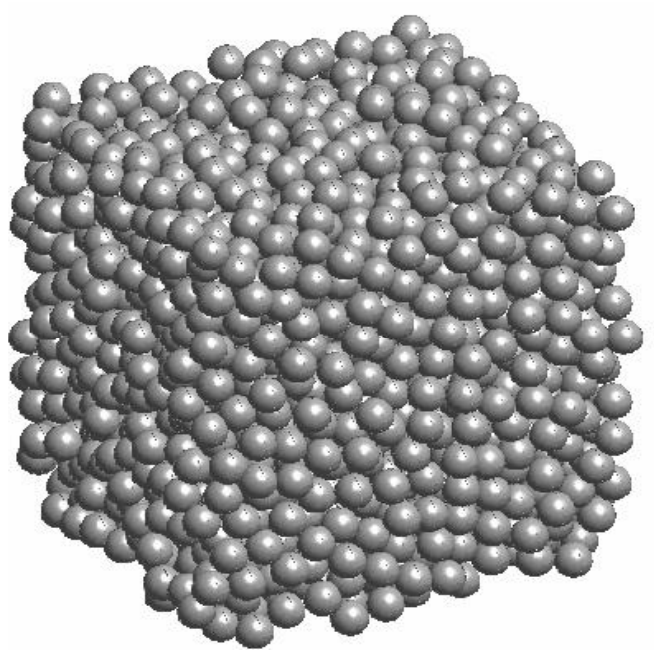

Figure 6: A random packing of balls obtained by the force based algorithm. This configuration consists of 4096 balls of constant radius $r=0.0332$, their centers are sited in the unit cube. The sample satisfies periodic boundary conditions.

From the crystallographic point of view the interpretation is as follows: The positions of the maxima of the density of the Bartlett spectrum are identical with those of the hexagonal dense packing (hdp) of balls where the balls' centers form an hdp-lattice. The positions of the interferences of the [100]-, [002]-, and [220]-planes of the hdplattice are marked in Figure $7 \mathrm{~b}$ where $[h k l]$ denote the Laue indices of lattice planes, 


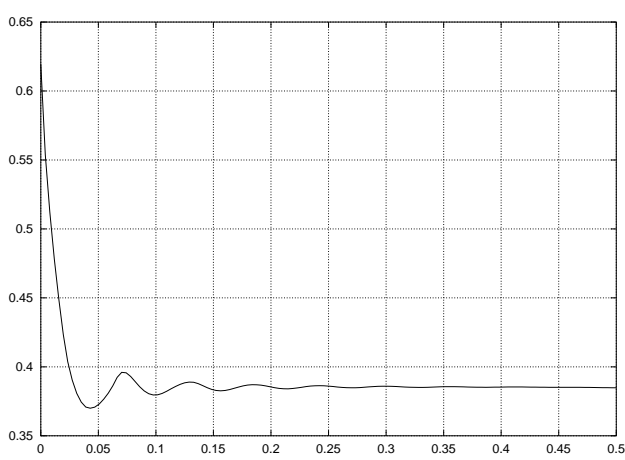

(a) Covariance

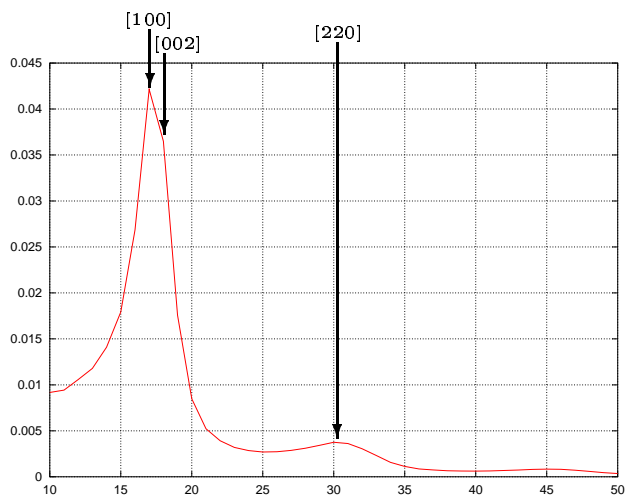

(b) Density of the Bartlett spectrum

Figure 7: (a) Rotation mean of the estimated covariance and (b) the density of the Bartlett spectrum of the dense packing of balls shown in Figure 6 .

see e.g. [11]. Obviously, there is a tendency of this random packing to form a hexagonal dense packing. The random packing can be considered as a highly disturbed hdp.

\section{Acknowledgement}

The authors are grateful to the Deutsche Forschungsgemeinschaft (Oh 59/5-1) for financial support. We thank S. Gondrom for scanning the nickel foam (Figure 4) and E. Ziegler for generating the visualization of the dense packing (Figure 6).

\section{References}

[1] Bartlett, M. (1963). The spectral analysis of point processes. J. R. Statist. Soc. B 29, 264-296.

[2] Bartlett, M. (1964). The spectral analysis of two-dimensional point processes. Biometrika 51, 299-311.

[3] Berke, A., Neite, G., Riehemann, W. And Nembach, E. (1987). Characterization of periodic composites by laser-beam diffraction. J. Appl. Phys. 61, $1263-1267$.

[4] Böhm, S., Heinrich, L. And Schmidt, V. (2002). Kernel estimation of the spectral density of stationary random closed sets. Preprint. 
[5] Brigham, E. (1995). FFT: Schnelle Fourier-Transformation 6. ed. Oldenbourg, München-Wien.

[6] Daley, D. and Vere-Jones, D. (1988). An Introduction to the Theory of Point Processes. Springer, New York.

[7] Debye, P., Anderson, H. R. and Brumberger, H. (1957). Scattering by an inhomogeneous solid. II. The correlation function and its application. Journal of Applied Physics 28, 679-683.

[8] Dreseler, B. ANd Schempp, W. (1980). Einführung in die harmonische Analyse. Teubner, Stuttgart.

[9] Frank, J. (1980). The role of correlation techniques in computer image processing. In Computer Processing of Electron Microscope Images. ed. P. Hawkes. Springer, Berlin. pp. 187-222.

[10] Frigo, M. And Johnson, S. G. FFTW 2.1.3 (the fastest Fourier transform of the West). http://www.fftw.org/ 1998.

[11] Henry, N. F. M. And Lonsdale, K. (1969). Internationl tables for X-ray crystallography vol. 1. Kynoch, Birmingham.

[12] KосH, K. (2002). Spektralanalyse zufälliger abgeschlossener Mengen. Master's thesis. University of Siegen.

[13] Manolakis, D. and Proakis, J. (1996). Digital Signal Processing 3. ed. Prentice-Hall.

[14] Marcotte, D. (1996). Fast variogram computation with fft. Computers \& Geosciences 22, 1175-1186

[15] Mugglestone, M. And Renshaw, E. (1996). A practical guide to the spectral analysis of spatial point processes. Computational Statistics \& Data Analysis 21, $43-65$.

[16] OhSER, J. AND MÜCKLICh, F. (2000). Statistical Analysis of Microstructures in Materials Science. Wiley, Chichester. 
[17] Redon, C., Chermant, L., Chermant, J.-L. and Coster, M. (1998). Assessment of fibre orientation in reinforced concrete using fourier image transform. Journal of Microscopy 191, 258-265.

[18] Renshaw, E. and Ford, E. (1983). The interpretation of process from pattern using two-dimensional spectral analysis: Methods and problems of interpretation. Applied Statistics 32, 51-63.

[19] Ripley, B. (1981). Spatial Statistics. Wiley, New York.

[20] Sasvari, Z. (1994). Positive Definite and Definitizable Functions. Akademie Verlag, Berlin.

[21] Schneider, R. And Weil, W. (2000). Stochastische Geometrie. Teubner, Stuttgart-Leipzig.

[22] Stoyan, D. (2002). Random systems of hard particles: models and statistics. Chinese J. Stereology and Image Analysis 7, 1-14.

[23] Stoyan, D., Kendall, W. and Mecke, J. (1995). Stochastic Geometry and its Applications 2. ed. Wiley, Chichester.

[24] Vurpillot, F., Da Costa, G., Menand, A. and Blavette, D. (2001). Structural analysis in three-dimensional atom probe: a Fourier transform approach. Journal of Microscopy 203, 295-302. 


\author{
Die PDF-Files der folgenden Berichte \\ finden Sie unter: \\ www.itwm.fhg.de/zentral/berichte.html
}

1. D. Hietel, K. Steiner, J. Struckmeier

\section{A Finite - Volume Particle Method for} Compressible Flows

We derive a new class of particle methods for conservation laws, which are based on numerical flux functions to model the interactions between moving particles. The derivation is similar to that of classical Finite-Volume methods; except that the fixed grid structure in the Finite-Volume method is substituted by so-called mass packets of particles. We give some numerical results on a shock wave solution for Burgers equation as well as the well-known one-dimensional shock tube problem. (19 S., 1998)

\section{M. Feldmann, S. Seibold}

\section{Damage Diagnosis of Rotors: Application of Hilbert Transform and Multi-Hypothesis Testing}

In this paper, a combined approach to damage diagnosis of rotors is proposed. The intention is to employ signalbased as well as model-based procedures for an improved detection of size and location of the damage. In a first step, Hilbert transform signal processing techniques allow for a computation of the signal envelope and the instantaneous frequency, so that various types of nonlinearities due to a damage may be identified and classified based on measured response data. In a second step, a multi-hypothesis bank of Kalman Filters is employed for the detection of the size and location of the damage based on the information of the type of damage provided by the results of the Hilbert transform.

Keywords:

Hilbert transform, damage diagnosis, Kalman filtering, non-linear dynamics

(23 S., 1998)

\section{Y. Ben-Haim, S. Seibold}

\section{Robust Reliability of Diagnostic Multi- Hypothesis Algorithms: Application to Rotating Machinery}

Damage diagnosis based on a bank of Kalman filters, each one conditioned on a specific hypothesized system condition, is a well recognized and powerful diagnostic tool. This multi-hypothesis approach can be applied to a wide range of damage conditions. In this paper, we will focus on the diagnosis of cracks in rotating machinery. The question we address is: how to optimize the multihypothesis algorithm with respect to the uncertainty of the spatial form and location of cracks and their resulting dynamic effects. First, we formulate a measure of the reliability of the diagnostic algorithm, and then we discuss modifications of the diagnostic algorithm for the maximization of the reliability. The reliability of a diagnostic algorithm is measured by the amount of uncertainty consistent with no-failure of the diagnosis. Uncertainty is quantitatively represented with convex models. Keywords:

Robust reliability, convex models, Kalman filtering, multihypothesis diagnosis, rotating machinery, crack diagnosis (24 S., 1998)
4. F.-Th. Lentes, N. Siedow

Three-dimensional Radiative Heat Transfer in Glass Cooling Processes

For the numerical simulation of 3D radiative heat transfer in glasses and glass melts, practically applicable mathematical methods are needed to handle such problems optimal using workstation class computers. Since the exact solution would require super-computer capabilities we concentrate on approximate solutions with a high degree of accuracy. The following approaches are studied: 3D diffusion approximations and 3D ray-tracing methods

(23 S., 1998)

\section{A. Klar, R. Wegener}

\section{A hierarchy of models for multilane vehicular traffic Part l: Modeling}

In the present paper multilane models for vehicular traffic are considered. A microscopic multilane model based on reaction thresholds is developed. Based on this model an Enskog like kinetic model is developed. In particular, care is taken to incorporate the correlations between the vehicles. From the kinetic model a fluid dynamic model is derived. The macroscopic coefficients are deduced from the underlying kinetic model. Numerical simulations are presented for all three levels of description in [10]. Moreover, a comparison of the results is given there. (23 S., 1998)

\section{Part II: Numerical and stochastic investigations}

In this paper the work presented in [6] is continued. The present paper contains detailed numerical investigations of the models developed there. A numerical method to treat the kinetic equations obtained in [6] are presented and results of the simulations are shown. Moreover, the stochastic correlation model used in [6] is described and investigated in more detail.

(17 S., 1998)

\section{A. Klar, N. Siedow}

Boundary Layers and Domain Decomposition for Radiative Heat Transfer and Diffusion Equations: Applications to Glass Manufacturing Processes

In this paper domain decomposition methods for radiative transfer problems including conductive heat transfer are treated. The paper focuses on semi-transparent materials, like glass, and the associated conditions at the interface between the materials. Using asymptotic analysis we derive conditions for the coupling of the radiative transfer equations and a diffusion approximation. Several test cases are treated and a problem appearing in glass manufacturing processes is computed. The results clearly show the advantages of a domain decomposition ap proach. Accuracy equivalent to the solution of the global radiative transfer solution is achieved, whereas computation time is strongly reduced.

(24 S., 1998)

\section{I. Choquet}

Heterogeneous catalysis modelling and numerical simulation in rarified gas flows Part I: Coverage locally at equilibrium

A new approach is proposed to model and simulate numerically heterogeneous catalysis in rarefied gas flows. It is developed to satisfy all together the following points: 1) describe the gas phase at the microscopic scale, as required in rarefied flows,

2) describe the wall at the macroscopic scale, to avoid prohibitive computational costs and consider not only crystalline but also amorphous surfaces,

3) reproduce on average macroscopic laws correlated with experimental results and

4) derive analytic models in a systematic and exact way. The problem is stated in the general framework of a non static flow in the vicinity of a catalytic and non porous surface (without aging). It is shown that the exact and systematic resolution method based on the Laplace transform, introduced previously by the author to model collisions in the gas phase, can be extended to the present problem. The proposed approach is applied to the modelling of the Eley-Rideal and Langmuir-Hinshelwood recombinations, assuming that the coverage is locally at equilibrium. The models are developed considering one atomic species and extended to the general case of several atomic species. Numerical calculations show that the models derived in this way reproduce with accuracy behaviors observed experimentally.

(24 S., 1998)

\section{J. Ohser, B. Steinbach, C. Lang}

\section{Efficient Texture Analysis of Binary Images}

A new method of determining some characteristics of binary images is proposed based on a special linear filtering. This technique enables the estimation of the area fraction, the specific line length, and the specific integral of curvature. Furthermore, the specific length of the tota projection is obtained, which gives detailed information about the texture of the image. The influence of lateral and directional resolution depending on the size of the applied filter mask is discussed in detail. The technique includes a method of increasing directional resolution for texture analysis while keeping lateral resolution as high as possible.

(17 S., 1998)

\section{J. Orlik}

\section{Homogenization for viscoelasticity of the} integral type with aging and shrinkage

A multi-phase composite with periodic distributed inclusions with a smooth boundary is considered in this contribution. The composite component materials are supposed to be linear viscoelastic and aging (of the non-convolution integral type, for which the Laplace transform with respect to time is not effectively applicable) and are subjected to isotropic shrinkage. The free shrinkage deformation can be considered as a fictitious temperature deformation in the behavior law. The procedure presented in this paper proposes a way to determine average (effective homogenized) viscoelastic and shrinkage (temperature) composite properties and the homogenized stress-field from known properties of the 
components. This is done by the extension of the asymptotic homogenization technique known for pure elastic non-homogeneous bodies to the non-homogeneous thermo-viscoelasticity of the integral non-convolution type. Up to now, the homogenization theory has not covered viscoelasticity of the integral type.

Sanchez-Palencia (1980), Francfort \& Suquet (1987) (see [2], [9]) have considered homogenization for viscoelasticity of the differential form and only up to the first derivative order. The integral-modeled viscoelasticity is more general then the differential one and includes almost all known differential models. The homogenization procedure is based on the construction of an asymptotic solution with respect to a period of the composite structure. This reduces the original problem to some auxiliary boundary value problems of elasticity and viscoelasticity on the unit periodic cell, of the same type as the original non-homogeneous problem. The existence and uniqueness results for such problems were obtained for kernels satisfying some constrain conditions. This is done by the extension of the Volterra integral operator theory to the Volterra operators with respect to the time, whose 1 kernels are space linear operators for any fixed time variables. Some ideas of such approach were proposed in [11] and [12], where the Volterra operators with kernels depending additionally on parameter were considered. This manuscript delivers results of the same nature for the case of the space-operator kernels.

(20 S., 1998)

\section{J. Mohring}

\section{Helmholtz Resonators with Large Aperture}

The lowest resonant frequency of a cavity resonator is usually approximated by the classical Helmholtz formula. However, if the opening is rather large and the front wal is narrow this formula is no longer valid. Here we present a correction which is of third order in the ratio of the diameters of aperture and cavity. In addition to the high accuracy it allows to estimate the damping due to radiation. The result is found by applying the method of matched asymptotic expansions. The correction contains form factors describing the shapes of opening and cavity. They are computed for a number of standard geometries. Results are compared with numerical computations. (21 S., 1998)

\section{H. W. Hamacher, A. Schöbel \\ On Center Cycles in Grid Graphs}

Finding "good" cycles in graphs is a problem of great interest in graph theory as well as in locational analysis. We show that the center and median problems are NP hard in general graphs. This result holds both for the vari able cardinality case (i.e. all cycles of the graph are considered) and the fixed cardinality case (i.e. only cycles with a given cardinality $p$ are feasible). Hence it is of interest to investigate special cases where the problem is solvable in polynomial time.

In grid graphs, the variable cardinality case is, for instance, trivially solvable if the shape of the cycle can be chosen freely.

If the shape is fixed to be a rectangle one can analyze rectangles in grid graphs with, in sequence, fixed dimension, fixed cardinality, and variable cardinality. In all cases a complete characterization of the optimal cycles and closed form expressions of the optimal objective values are given, yielding polynomial time algorithms for all cases of center rectangle problems.

Finally, it is shown that center cycles can be chosen as rectangles for small cardinalities such that the center cycle problem in grid graphs is in these cases completely solved.

(15 S., 1998)

\section{H. W. Hamacher, K.-H. Küfer}

\section{Inverse radiation therapy planning -} a multiple objective optimisation approach

For some decades radiation therapy has been proved successful in cancer treatment. It is the major task of clinical radiation treatment planning to realize on the one hand a high level dose of radiation in the cancer tissue in order to obtain maximum tumor control. On the other hand it is obvious that it is absolutely necessary to keep in the tissue outside the tumor, particularly in organs at risk, the unavoidable radiation as low as possible. No doubt, these two objectives of treatment planning high level dose in the tumor, low radiation outside the tumor - have a basically contradictory nature. Therefore, it is no surprise that inverse mathematical models with dose distribution bounds tend to be infeasible in most cases. Thus, there is need for approximations compromising between overdosing the organs at risk and underdosing the target volume.

Differing from the currently used time consuming iterative approach, which measures deviation from an ideal (non-achievable) treatment plan using recursively trialand-error weights for the organs of interest, we go a new way trying to avoid a priori weight choices and consider the treatment planning problem as a multiple objective linear programming problem: with each organ of interest, target tissue as well as organs at risk, we associate an objective function measuring the maximal deviation from the prescribed doses.

We build up a data base of relatively few efficient solutions representing and approximating the variety of Pareto solutions of the multiple objective linear programming problem. This data base can be easily scanned by physicians looking for an adequate treatment plan with the aid of an appropriate online tool.

(14 S., 1999)

\section{C. Lang, J. Ohser, R. Hilfer}

\section{On the Analysis of Spatial Binary Images}

This paper deals with the characterization of microscopically heterogeneous, but macroscopically homogeneous spatial structures. A new method is presented which is strictly based on integral-geometric formulae such as Crofton's intersection formulae and Hadwiger's recursive definition of the Euler number. The corresponding algorithms have clear advantages over other techniques. As an example of application we consider the analysis of spatial digital images produced by means of Computer Assisted Tomography.

(20 S., 1999)

\section{M. Junk}

\section{On the Construction of Discrete Equilibrium} Distributions for Kinetic Schemes

A general approach to the construction of discrete equilibrium distributions is presented. Such distribution func tions can be used to set up Kinetic Schemes as well as Lattice Boltzmann methods. The general principles are also applied to the construction of Chapman Enskog distributions which are used in Kinetic Schemes for com- pressible Navier-Stokes equations. (24 S., 1999)

\section{M. Junk, S. V. Raghurame Rao}

\section{A new discrete velocity method for Navier Stokes equations}

The relation between the Lattice Boltzmann Method which has recently become popular, and the Kinetic Schemes, which are routinely used in Computational Fluid Dynamics, is explored. A new discrete velocity mode for the numerical solution of Navier-Stokes equations for incompressible fluid flow is presented by combining both the approaches. The new scheme can be interpreted as a pseudo-compressibility method and, for a particular choice of parameters, this interpretation carries over to the Lattice Boltzmann Method. (20 S., 1999)

\section{H. Neunzert}

\section{Mathematics as a Key to Key Technologies}

The main part of this paper will consist of examples, how mathematics really helps to solve industrial problems; these examples are taken from our Institute for Industrial Mathematics, from research in the Technomathematics group at my university, but also from ECMI groups and a company called TecMath, which originated 10 years ago from my university group and has already a very successful history.

39 S. (vier PDF-Files), 1999)

\section{J. Ohser, K. Sandau}

\section{Considerations about the Estimation of the} Size Distribution in Wicksell's Corpuscle Problem

Wicksell's corpuscle problem deals with the estimation of the size distribution of a population of particles, all having the same shape, using a lower dimensional sampling probe. This problem was originary formulated for particle systems occurring in life sciences but its solution is of actual and increasing interest in materials science. From a mathematical point of view, Wicksell's problem is an inverse problem where the interesting size distribution is the unknown part of a Volterra equation. The problem is often regarded ill-posed, because the structure of the integrand implies unstable numerical solutions. The accuracy of the numerical solutions is considered here using the condition number, which allows to compare different numerical methods with different (equidistant) class sizes and which indicates, as one result, that a finite section thickness of the probe reduces the numerical problems. Furthermore, the relative error of estimation is computed which can be split into two parts. One part consists of the relative discretization error that increases for increasing class size, and the second part is related to the relative statistical error which increases with decreasing class size. For both parts, upper bounds can be given and the sum of them indicates an optimal class width depending on some specific constants.

(18 S., 1999) 
18. E. Carrizosa, H. W. Hamacher, R. Klein, S. Nickel

\section{Solving nonconvex planar location problems by finite dominating sets}

It is well-known that some of the classical location problems with polyhedral gauges can be solved in polynomia time by finding a finite dominating set, i. e. a finite set of candidates guaranteed to contain at least one optimal location.

In this paper it is first established that this result holds for a much larger class of problems than currently considered in the literature. The model for which this result can be proven includes, for instance, location problems with attraction and repulsion, and location-allocation problems. Next, it is shown that the approximation of general gauges by polyhedral ones in the objective function of our general model can be analyzed with regard to the subsequent error in the optimal objective value. For the approx imation problem two different approaches are described, the sandwich procedure and the greedy algorithm. Both of these approaches lead - for fixed epsilon - to polynomial approximation algorithms with accuracy epsilon for solving the general model considered in this paper. Keywords:

Continuous Location, Polyhedral Gauges, Finite Dominating Sets, Approximation, Sandwich Algorithm, Greedy Algorithm

(19 S., 2000)

\section{A. Becker}

\section{A Review on Image Distortion Measures}

Within this paper we review image distortion measures. A distortion measure is a criterion that assigns a "quality number" to an image. We distinguish between mathematical distortion measures and those distortion measures in-cooperating a priori knowledge about the imaging devices (e. g. satellite images), image processing algorithms or the human physiology. We will consider representative examples of different kinds of distortion measures and are going to discuss them. Keywords:

Distortion measure, human visual system (26 S., 2000)

\section{H. W. Hamacher, M. Labbé, S. Nickel} T. Sonneborn

\section{Polyhedral Properties of the Uncapacitated Multiple Allocation Hub Location Problem}

We examine the feasibility polyhedron of the uncapacitated hub location problem (UHL) with multiple allocation, which has applications in the fields of air passenger and cargo transportation, telecommunication and postal delivery services. In particular we determine the dimension and derive some classes of facets of this polyhedron. We develop some general rules about lifting facets from the uncapacitated facility location (UFL) for UHL and projecting facets from UHL to UFL. By applying these rules we get a new class of facets for UHL which dominates the inequalities in the original formulation. Thus we get a new formulation of UHL whose constraints are all facetdefining. We show its superior computational performance by benchmarking it on a well known data set Keywords:

integer programming, hub location, facility location, valid inequalities, facets, branch and cut

(21 S., 2000)

\section{H. W. Hamacher, A. Schöbel \\ Design of Zone Tariff Systems in Public Transportation}

Given a public transportation system represented by its stops and direct connections between stops, we consider two problems dealing with the prices for the customers: The fare problem in which subsets of stops are already aggregated to zones and "good" tariffs have to be found in the existing zone system. Closed form solutions for the fare problem are presented for three objective functions. In the zone problem the design of the zones is part of the problem. This problem is NP hard and we therefore propose three heuristics which prove to be very successful in the redesign of one of Germany's transportation systems. (30 S., 2001)

\section{D. Hietel, M. Junk, R. Keck, D. Teleaga: \\ The Finite-Volume-Particle Method for Conservation Laws}

In the Finite-Volume-Particle Method (FVPM), the weak formulation of a hyperbolic conservation law is discretized by restricting it to a discrete set of test functions. In contrast to the usual Finite-Volume approach, the test functions are not taken as characteristic functions of the control volumes in a spatial grid, but are chosen from a partition of unity with smooth and overlapping partition functions (the particles), which can even move along prescribed velocity fields. The information exchange between particles is based on standard numerical flux functions. Geometrical information, similar to the surface area of the cell faces in the Finite-Volume Method and the corresponding normal directions are given as integral quantities of the partition functions.

After a brief derivation of the Finite-Volume-Particle Method, this work focuses on the role of the geometric coefficients in the scheme.

(16 S., 2001)

23. T. Bender, H. Hennes, J. Kalcsics,

M. T. Melo, S. Nickel

\section{Location Software and Interface with GIS and Supply Chain Management}

The objective of this paper is to bridge the gap between location theory and practice. To meet this objective focus is given to the development of software capable of addressing the different needs of a wide group of users. There is a very active community on location theory encompassing many research fields such as operations research, computer science, mathematics, engineering, geography, economics and marketing. As a result, people working on facility location problems have a very diverse background and also different needs regarding the software to solve these problems. For those interested in non-commercial applications (e. g. students and researchers), the library of location algorithms (LoLA can be of considerable assistance. LoLA contains a collection of efficient algorithms for solving planar, network and discrete facility location problems. In this paper, a detailed description of the functionality of LoLA is presented. In the fields of geography and marketing, for instance, solving facility location problems requires using large amounts of demographic data. Hence, members of these groups (e. g. urban planners and sales managers) often work with geographical information too $\mathrm{s}$. To address the specific needs of these users, LoLA was inked to a geo- graphical information system (GIS) and the details of the combined functionality are described in the paper. Finally, there is a wide group of practitioners who need to solve large problems and require special purpose software with a good data interface. Many of such users can be found for example, in the area of supply chain management (SCM). Logistics activities involved in strategic SCM include, among others, facility location planning. In this paper, the development of a commercial location software tool is also described. The too is embedded in the Advanced Planner and Optimizer SCM software developed by SAP AG, Walldorf, Germany. The paper ends with some conclusions and an outlook to future activities.

Keywords:

facility location, software development, geographical information systems, supply chain management. (48 S., 2001)

\section{H. W. Hamacher, S. A. Tjandra Mathematical Modelling of Evacuation Problems: A State of Art}

This paper details models and algorithms which can be applied to evacuation problems. While it concentrates on building evacuation many of the results are applicable also to regional evacuation. All models consider the time as main parameter, where the travel time between components of the building is part of the input and the overall evacuation time is the output. The paper distinguishes between macroscopic and microscopic evacuation models both of which are able to capture the evacuees' movement over time.

Macroscopic models are mainly used to produce good lower bounds for the evacuation time and do not consider any individual behavior during the emergency situation. These bounds can be used to analyze existing buildings or help in the design phase of planning a building Macroscopic approaches which are based on dynamic network flow models (minimum cost dynamic flow, maximum dynamic flow, universal maximum flow, quickest path and quickest flow) are described. A special feature of the presented approach is the fact, that travel times of evacuees are not restricted to be constant, but may be density dependent. Using multicriteria optimization priority regions and blockage due to fire or smoke may be considered. It is shown how the modelling can be done using time parameter either as discrete or continuous parameter

Microscopic models are able to model the individual evacuee's characteristics and the interaction among evacuees which influence their movement. Due to the corresponding huge amount of data one uses simulation approaches. Some probabilistic laws for individual evacuee's movement are presented. Moreover ideas to model the evacuee's movement using cellular automata (CA) and resulting software are presented.

In this paper we will focus on macroscopic models and only summarize some of the results of the microscopic approach. While most of the results are applicable to general evacuation situations, we concentrate on building evacuation.

(44 S., 2001) 
25. J. Kuhnert, S. Tiwari

\section{Grid free method for solving the Poisson equation}

A Grid free method for solving the Poisson equation is presented. This is an iterative method. The method is based on the weighted least squares approximation in which the Poisson equation is enforced to be satisfied in every iterations. The boundary conditions can also be enforced in the iteration process. This is a local approximation procedure. The Dirichlet, Neumann and mixed boundary value problems on a unit square are presented and the analytical solutions are compared with the exact solutions. Both solutions matched perfectly. Keywords:

Poisson equation, Least squares method, Grid free method (19 S., 2001)

26. T. Götz, H. Rave, D. Reinel-Bitzer, K. Steiner, H. Tiemeier

\section{Simulation of the fiber spinning process}

To simulate the influence of process parameters to the melt spinning process a fiber model is used and coupled with CFD calculations of the quench air flow. In the fiber model energy, momentum and mass balance are solved for the polymer mass flow. To calculate the quench air the Lattice Boltzmann method is used. Simulations and experiments for different process parameters and hole configurations are compared and show a good agreement.

Keywords:

Melt spinning, fiber model, Lattice Boltzmann, CFD (19 S., 2001)

\section{A. Zemitis}

\section{On interaction of a liquid film with an obstacle}

In this paper mathematical models for liquid films generated by impinging jets are discussed. Attention is stressed to the interaction of the liquid film with some obstacle. S. G. Taylor [Proc. R. Soc. London Ser. A 253, 313 (1959)] found that the liquid film generated by impinging jets is very sensitive to properties of the wire which was used as an obstacle. The aim of this presentation is to propose a modification of the Taylor's model, which allows to simulate the film shape in cases, when the angle between jets is different from $180^{\circ}$. Numerical results obtained by discussed models give two different shapes of the liquid film similar as in Taylors experiments. These two shapes depend on the regime: either droplets are produced close to the obstacle or not. The difference between two regimes becomes larger if the angle between jets decreases. Existence of such two regimes can be very essential for some applications of impinging jets, if the generated liquid film can have a contact with obstacles. Keywords:

impinging jets, liquid film, models, numerical solution, shape

(22 S., 2001)

\section{I. Ginzburg, K. Steiner}

\section{Free surface lattice-Boltzmann method to model the filling of expanding cavities by Bingham Fluids}

The filling process of viscoplastic metal alloys and plastics in expanding cavities is modelled using the lattice Boltzmann method in two and three dimensions. These mod els combine the regularized Bingham model for viscoplastic with a free-interface algorithm. The latter is based on a modified immiscible lattice Boltzmann model in which one species is the fluid and the other one is considered as vacuum. The boundary conditions at the curved liquid-vacuum interface are met without any geometrical front reconstruction from a first-order ChapmanEnskog expansion. The numerical results obtained with these models are found in good agreement with available theoretical and numerical analysis. Keywords:

Generalized LBE, free-surface phenomena, interface boundary conditions, filling processes, Bingham viscoplastic model, regularized models (22 S., 2001)

\section{H. Neunzert}

"Denn nichts ist für den Menschen als Menschen etwas wert, was er nicht mit Leidenschaft tun kann"

Vortrag anlässlich der Verleihung des Akademiepreises des Landes Rheinland-Pfalz am

\subsubsection{1}

Was macht einen guten Hochschullehrer aus? Auf diese Frage gibt es sicher viele verschiedene, fachbezogene Antworten, aber auch ein paar allgemeine Gesichtspunkte: es bedarf der »Leidenschaft « für die Forschung (Max Weber), aus der dann auch die Begeisterung für die Lehre erwächst. Forschung und Lehre gehören zusammen, um die Wissenschaft als lebendiges Tun vermitteln zu können. Der Vortrag gibt Beispiele dafür, wie in angewandter Mathematik Forschungsaufgaben aus praktischen Alltagsproblemstellungen erwachsen, die in die Lehre auf verschiedenen Stufen (Gymnasium bis Graduiertenkolleg) einfließen; er leitet damit auch zu einem aktuellen Forschungsgebiet, der Mehrskalenanalyse mit ihren vielfältigen Anwendungen in Bildverarbeitung Materialentwicklung und Strömungsmechanik über, was aber nur kurz gestreift wird. Mathematik erscheint hier als eine moderne Schlüsseltechnologie, die aber auch enge Beziehungen zu den Geistes- und Sozialwissenschaften hat.

Keywords:

Lehre, Forschung, angewandte Mathematik, Mehrskalenanalyse, Strömungsmechanik

(18 S., 2001)

30. J. Kuhnert, S. Tiwari

Finite pointset method based on the projection method for simulations of the incom pressible Navier-Stokes equations

A Lagrangian particle scheme is applied to the projection method for the incompressible Navier-Stokes equations. The approximation of spatial derivatives is obtained by the weighted least squares method. The pressure Poisson equation is solved by a local iterative procedure with the help of the least squares method. Numerical tests are performed for two dimensional cases. The Couette flow Poiseuelle flow, decaying shear flow and the driven cavity flow are presented. The numerical solutions are obtained for stationary as well as instationary cases and are compared with the analytical solutions for channel flows. Finally, the driven cavity in a unit square is considered and the stationary solution obtained from this scheme is compared with that from the finite element method. Keywords:

Incompressible Navier-Stokes equations, Meshfree method, Projection method, Particle scheme, Least squares approximation

AMS subject classification:

76D05, 76M28

(25 S., 2001)

\section{R. Korn, M. Krekel Optimal Portfolios with Fixed Consumption or Income Streams}

We consider some portfolio optimisation problems where either the investor has a desire for an a priori specified consumption stream or/and follows a deterministic pay in scheme while also trying to maximize expected utility from final wealth. We derive explicit closed form solutions for continuous and discrete monetary streams. The mathematical method used is classical stochastic control theory.

Keywords:

Portfolio optimisation, stochastic control, HJB equation, discretisation of control problems.

(23 S., 2002)

\section{M. Krekel}

\section{Optimal portfolios with a loan dependent credit spread}

If an investor borrows money he generally has to pay higher interest rates than he would have received, if he had put his funds on a savings account. The classical model of continuous time portfolio optimisation ignores this effect. Since there is obviously a connection between the default probability and the total percentage of wealth, which the investor is in debt, we study portfolio optimisation with a control dependent interest rate. Assuming a logarithmic and a power utility function, respectively, we prove explicit formulae of the optimal control.

Keywords:

Portfolio optimisation, stochastic control, HJB equation, credit spread, log utility, power utility, non-linear wealth dynamics

(25 S., 2002)

\section{J. Ohser, W. Nagel, K. Schladitz}

The Euler number of discretized sets - on the choice of adjacency in homogeneous lattices

Two approaches for determining the Euler-Poincaré characteristic of a set observed on lattice points are considered in the context of image analysis \{ the integral geometric and the polyhedral approach. Information about the set is assumed to be available on lattice points only. In order to retain properties of the Euler number and to provide a good approximation of the true Euler number of the original set in the Euclidean space, the appropriate choice of adjacency in the lattice for the set and its background is crucial. Adjacencies are defined using tessellations of the whole space into polyhedrons. In $\mathrm{R} 3$ two new 14 adjacencies are introduced additionally to 
the well known 6 and 26 adjacencies. For the Euler number of a set and its complement, a consistency relation holds. Each of the pairs of adjacencies $(14: 1 ; 14: 1)$ $(14: 2 ; 14: 2),(6 ; 26)$, and $(26 ; 6)$ is shown to be a pair of complementary adjacencies with respect to this relation. That is, the approximations of the Euler numbers are consistent if the set and its background (complement) are equipped with this pair of adjacencies. Furthermore, sufficient conditions for the correctness of the approximations of the Euler number are given. The analysis of selected microstructures and a simulation study illustrate how the estimated Euler number depends on the chosen adjacency. It also shows that there is not a uniquely best pair of adjacencies with respect to the estimation of the Euler number of a set in Euclidean space. Keywords: image analysis, Euler number, neighborhod relationships, cuboidal lattice

(32 S., 2002)

\section{I. Ginzburg, K. Steiner}

\section{Lattice Boltzmann Model for Free-Surface flow and Its Application to Filling Process in Casting}

A generalized lattice Boltzmann model to simulate freesurface is constructed in both two and three dimensions. The proposed model satisfies the interfacial boundary conditions accurately. A distinctive feature of the model is that the collision processes is carried out only on the points occupied partially or fully by the fluid. To maintain a sharp interfacial front, the method includes an antidiffusion algorithm. The unknown distribution functions at the interfacial region are constructed according to the first order Chapman-Enskog analysis. The interfacial boundary conditions are satisfied exactly by the coefficients in the Chapman-Enskog expansion. The distribution functions are naturally expressed in the local interfacial coordinates. The macroscopic quantities at the interface are extracted from the least-square solutions of a locally linearized system obtained from the known distribution functions. The proposed method does not require any geometric front construction and is robust for any interfacial topology. Simulation results of realistic filling process are presented: rectangular cavity in two dimensions and Hammer box, Campbell box, Sheffield box, and Motorblock in three dimensions. To enhance the stability at high Reynolds numbers, various upwind-type schemes are developed. Free-slip and no-slip boundary conditions are also discussed.

Keywords: Lattice Boltzmann models; free-surface phenomena; interface boundary conditions; filling processes; injection molding; volume of fluid method; interface boundary conditions; advection-schemes; upwindschemes

(54 S., 2002)

35. M. Günther, A. Klar, T. Materne, R. Wegener Multivalued fundamental diagrams and stop and go waves for continuum traffic equations

In the present paper a kinetic model for vehicular traffic leading to multivalued fundamental diagrams is developed and investigated in detail. For this model phase transitions can appear depending on the local density and velocity of the flow. A derivation of associated macroscopic traffic equations from the kinetic equation is given. Moreover, numerical experiments show the appearance of stop and go waves for highway traffic with a bottleneck.
Keywords: traffic flow, macroscopic equations, kinetic derivation, multivalued fundamental diagram, stop and go waves, phase transitions

(25 S., 2002)

\section{S. Feldmann, P. Lang, D. Prätzel-Wolters}

\section{Parameter influence on the zeros of network} determinants

To a network $\mathrm{N}(q)$ with determinant $\mathrm{D}(s ; q)$ depending on a parameter vector $q \hat{\imath} R^{r}$ via identification of some of its vertices, a network $\mathrm{N}^{\wedge}(q)$ is assigned. The paper deals with procedures to find $\mathrm{N}^{\wedge}(q)$, such that its determinant $D^{\wedge}(s ; q)$ admits a factorization in the determinants of appropriate subnetworks, and with the estimation of the deviation of the zeros of $D^{\wedge}$ from the zeros of $D$. To solve the estimation problem state space methods are applied.

Keywords: Networks, Equicofactor matrix polynomials, Realization theory, Matrix perturbation theory (30 S., 2002)

\section{K. Koch, J. Ohser, K. Schladitz}

\section{Spectral theory for random closed sets and} estimating the covariance via frequency space

A spectral theory for stationary random closed sets is developed and provided with a sound mathematical basis. Definition and proof of existence of the Bartlett spectrum of a stationary random closed set as well as the proof of a Wiener-Khintchine theorem for the power spectrum are used to two ends: First, well known second order characteristics like the covariance can be estimated faster than usual via frequency space. Second, the Bartlett spectrum and the power spectrum can be used as second order characteristics in frequency space. Examples show, that in some cases information about the random closed set is easier to obtain from these characteristics in frequency space than from their real world counterparts.

Keywords: Random set, Bartlett spectrum, fast Fourier transform, power spectrum

(28 S., 2002) 